Témoigner Témoigner. Entre histoire et mémoire

Getuigen Revue pluridisciplinaire de la Fondation Auschwitz

122 | 2016

Révisionisme et négationisme

\title{
Pourquoi les négationnistes restent dangereux
}

Why negationists remain dangerous

Waarom negationisten gevaarlijk blijven

\section{Aline Sax}

Traducteur : Emilie Syssau

\section{(2) OpenEdition}

Journals

\section{Édition électronique}

URL : https://journals.openedition.org/temoigner/4140

DOI : 10.4000/temoigner.4140

ISSN : 2506-6390

Éditeur :

Éditions du Centre d'études et de documentation Mémoire d'Auschwitz, Éditions Kimé

Édition imprimée

Date de publication : 2 mai 2016

Pagination : 64-71

ISSN : 2031-4183

Référence électronique

Aline Sax, «Pourquoi les négationnistes restent dangereux », Témoigner. Entre histoire et mémoire [En ligne], 122 | 2016, mis en ligne le 30 septembre 2021, consulté le 03 janvier 2022. URL : http:// journals.openedition.org/temoigner/4140; DOI : https://doi.org/10.4000/temoigner.4140 


\section{Pourquoi les négationnistes restent dangereux}

$\rightarrow$ Aline Sax

Geheugen Collectief

(1) Vrielink, J., Van haat gesproken? Een rechtsantropologisch onderzoek naar de bestrijding van rasgerelateerde uitingsdelicten in België, Maklu, et contributions à Knack (par exemple Decoene, 12 février 2015), Joods Actueel (2011), etc. es négationnistes - ceux qui contestent la Shoah - ont longtemps pu diffuser leurs convictions sans être vraiment inquiétés. Pour fournir de la crédibilité à leurs convictions, ils recourent à un arsenal de stratégies qui visent à faire vaciller les vérités historiques relatives à la Shoah et à rendre leurs idées plausibles. Nous étudierons dans cet article les stratégies mises en œuvre par les négationnistes et le danger qu'elles représentent pour la science historique.

\section{UN FORUM POUR NÉGATIONNISTES?}

La contestation, minimalisation, justification ou approbation du génocide perpétré pendant la Seconde Guerre mondiale par le régime national-socialiste allemand sont condamnées par une loi en Belgique depuis le 23 mars 1995. Des lois similaires s'appliquent dans de nombreux autres pays (européens) comme l’Allemagne, l'Autriche, la France, la République tchèque, la Hongrie, les Pays-Bas, la Pologne, le Portugal, la Roumanie, la Slovaquie, la Suisse, etc. (Lechtholz-Zey). Le bien-fondé ou l'absurdité de cet arrêté - qui, tout en se prévalant de la loi contre le racisme, contient aussi une restriction de la liberté d'expression - fait régulièrement débat. Les tenants de la loi estiment que les négationnistes ne doivent pas bénéficier d'une tribune et soulignent l'intention annexe de nombreux négationnistes : légitimer le nazisme. Les opposants à la loi pensent que, loin de faire changer d'avis les négationnistes, elle ne fera que les conforter dans leur conviction d'une conspiration (juive). De plus, elle amènerait les négationnistes à agir clandestinement, compliquant toute résistance à leurs propos. Les opposants à la loi y voient aussi une violation de la liberté d'expression, qu'ils jugent plus importante. L'extension ou non de cette loi à tous les génocides a également ses partisans et ses détracteurs ${ }^{1}$. En Belgique, les débats n'ont encore conduit ni à l'abrogation ni à l'élargissement de la loi. La Grande-Bretagne, le Danemark et la Suède ont déjà rejeté à plusieurs reprises des projets de loi visant à condamner le négationnisme (Bilefski, 2007), et le négationnisme n'est pas non plus interdit aux États-Unis. Le premier amendement place en effet la liberté d'expression au-dessus de toute autre loi. Les négationnistes y ont ainsi une tribune bien plus ouverte qu'en Europe. Mais ici aussi ces lois se sont fait attendre relativement longtemps : les premiers négationnistes se déchaînent contre le consensus historique sur la Shoah depuis l'immédiat après-guerre. 


\section{RÉVISIONNISME ET NÉGATIONNISME}

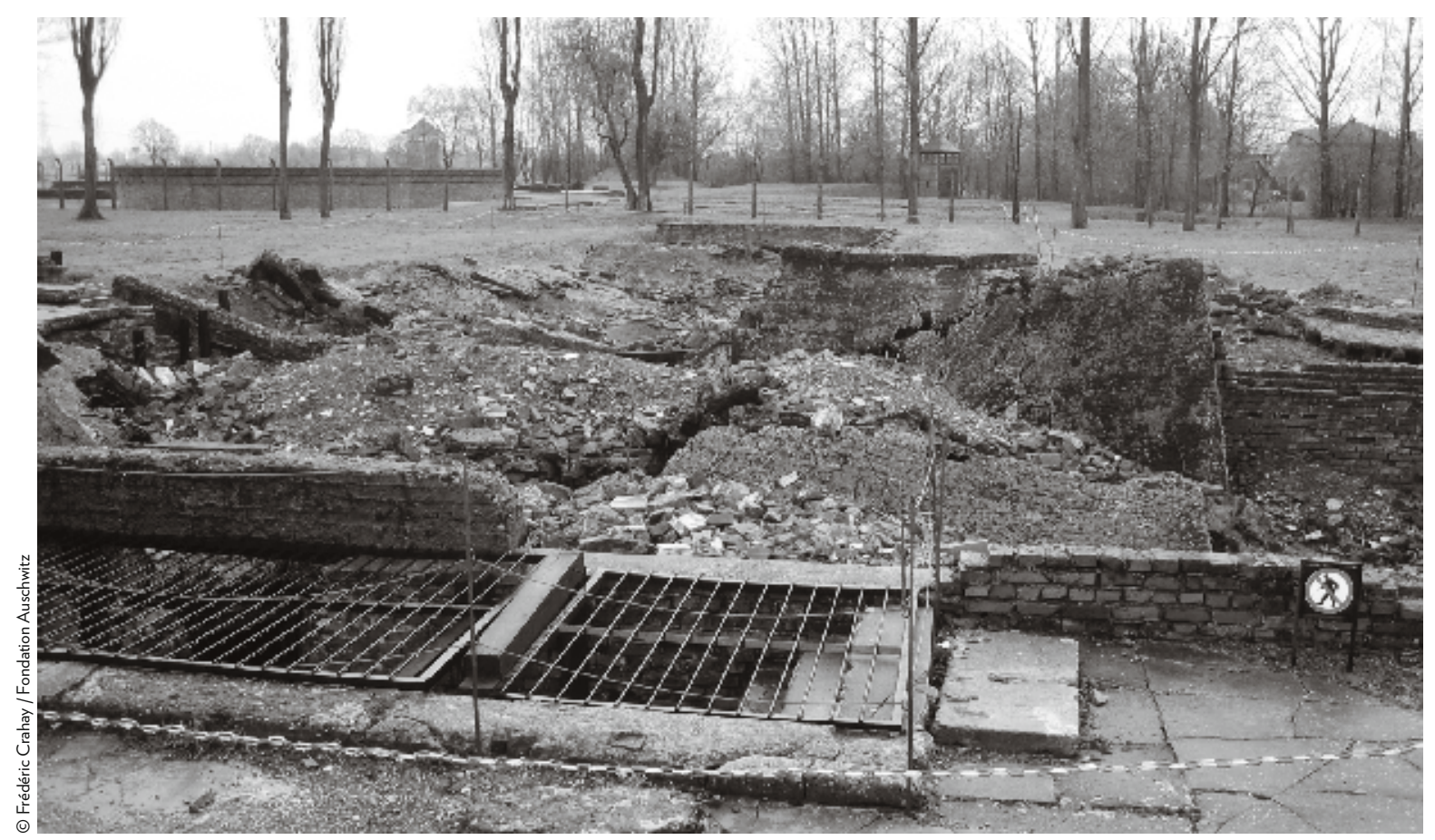

On trouve les premiers germes d'une pensée négationniste dès le procès de _Krematorium II à Birkenau. Nuremberg, qui apparaît à certains comme le procès des vainqueurs, en conséquence a priori inéquitable (Shermer \& Grobman, 2000, p. 40). Une allégation que les négationnistes n’ont cessé de citer au cours des décennies suivantes. Le premier négationniste influent a été le socialiste français Paul Rassinier. Il a lui-même été prisonnier à Buchenwald et Dora pour faits de résistance pendant la guerre. Après celle-ci, il a entendu des témoignages contradictoires et erronés, laissant supposer la présence de chambres à gaz à Buchenwald. Il les attribue à des mensonges intentionnels des témoins plutôt qu’à leur confusion. Convaincu que ces témoins ont menti, il met également en doute l'ampleur du génocide. En 1964, il publie le livre Le Drame des Juifs européens, dans lequel il affirme ce qui suit:

«En ce qui concerne les chambres à gaz : la procession pratiquement interminable de faux témoignages et de documents falsifiés [...] prouve une seule chose : que les autorités responsables du Troisième Reich n'ont à aucun moment eu l'intention d'exterminer les Juifs de quelque manière que ce soit. Cette extermination a-t-elle eu lieu sans que l'ordre en ait été donné ? Cette question me préoccupe depuis quinze ans. » (Widmann, 2010)

Le passé de prisonnier de Rassinier a bien sûr donné de la crédibilité à ses déclarations. La percée du négationnisme s'est faite surtout à partir des années 1960 et 1970, dans un véritable flot de publications (Shermer \& Grobman, 2000, p. 40-41). Les négationnistes ont alors diffusé leurs convictions comme des vérités aux ÉtatsUnis et en Europe. 


\section{DOSSIER}

Pourquoi les négationnistes restent dangereux (suite)
- Chambre à gaz du Krematorium III. Fred Leuchter y récolta (sans autorisation) des fragments en 1988 pour les analyser.
Le négationniste belge le plus connu est Siegfried Verbeke. Membre actif du VMO (Vlaamse Militanten Orde, Ordre des militants flamands) dans les années 1970, il imprime pour cette milice privée d'extrême droite toutes sortes de publications néonazies et antisémites. En 1985, il crée avec son frère l'association sans but lucratif (asbl) «Vrij Historisch Onderzoek» (Recherche historique libre), une organisation consacrée à la diffusion de pamphlets et publications négationnistes. «Vrij Historisch Onderzoek» fait surtout parler d'elle dans les années 1990, inondant de ses écrits, écoles, journalistes, politiques et historiens. Verbeke recherche à tort et à travers les projecteurs des médias pour diffuser son message. Le vote, en 1995, de la loi sur le négationnisme apporte de l'eau au moulin de Verbeke : cette loi prouve selon lui qu'il y a effectivement quelque chose à cacher. L'attention que les médias accordent à la loi joue également à son avantage : des poursuites judiciaires donneraient aux personnes poursuivies la possibilité d'exprimer leurs opinions négationnistes et ainsi de les propager. «Nous continuerons à contester et démasquer le génocide, les chambres à gaz et tout ce fatras de mensonges », écrit-il dans la lettre d'information de la VHO. Cela lui vaut plusieurs condamnations en Belgique, aux Pays-Bas et en Allemagne (Brinckman, 2005, p. 4; Timmerman, 2005, p. 4 et Vanermen, 1996, p. 126).

Mais à ces pamphlets se substitue progressivement un nouveau médium, beaucoup plus pratique : Internet. L’association continue à répandre ses idées négationnistes auprès de tous ceux qui prennent la peine de consulter son site Internet www.vho.org. Hébergé par des serveurs américains, ce site est hors d'atteinte du droit belge. Officiellement, Siegfried Verbeke a passé la main, mais le nom et l'adresse du site Internet renvoient toujours vers son association.

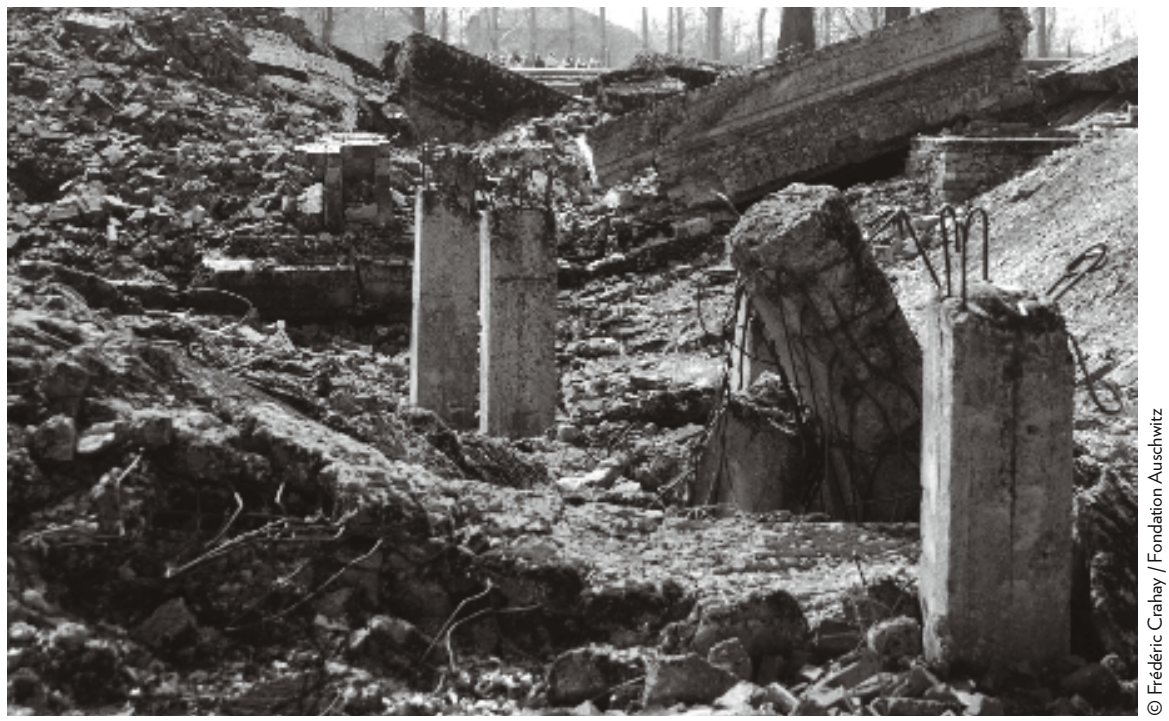




\section{CE QUE DISENT LES NÉGATIONNISTES}

Les opinions des négationnistes peuvent se résumer à grands traits en cinq pensées clés. Tout d'abord, la contestation de l'existence des chambres à gaz : selon eux, ces pièces n'étaient pas conçues pour assassiner des gens, mais pour désinfecter vêtements et bagages. Puis la négation du caractère méthodique et systématique de l'extermination des Juifs : la Endlösung (Solution finale) n'avait pas pour but d'exterminer les Juifs, seulement de les déplacer, et les camps étaient des camps temporaires de travail et de séjour, non des camps d'extermination pour assassiner les gens. Cette idée va de pair avec la contestation du nombre de six millions de morts : les négationnistes admettent que des Juifs sont morts dans les camps, mais affirment que ces décès sont dus aux conditions déplorables, comme la sousalimentation et les épidémies inévitablement liées au contexte de guerre; la Shoah n'était donc ni plus ni moins grave que les crimes de guerre perpétrés par les Alliés, comme les bombardements de Dresde et d'autres villes allemandes. Enfin, revient toujours la conviction d'une conspiration juive : la Shoah est une invention juive pour noircir Hitler et le nazisme, légitimer l'État d'Israël ou enrichir les Juifs ; les témoignages des camps sont des mensonges délibérés qu’il faut appréhender dans le cadre de cette conspiration (Shermer \& Grobman, 2000 ; Lipstadt, 1994 et Vanermen, 1996).

\section{STRATÉGIES}

Face aux preuves historiographiques étayant la Shoah comme l'extermination systématique de près de six millions de Juifs, il est difficile de croire que les négationnistes puissent imposer leur vision. Ils réussissent pourtant à semer systématiquement le doute et à attirer des gens de leur côté. Cela tient moins à ce qu'ils disent qu'aux stratégies utilisées pour remettre en question l'historiographie de la Shoah.

\section{UTILISER LE SAVOIR-FAIRE HISTORIQUE}

Les négationnistes essaient de mettre la science historique hors-jeu en appliquant beaucoup trop strictement les règles du jeu de cette science. Les publications ou déclarations négationnistes ne parlent jamais de «négationnisme», mais de «révisionnisme». Dans la forme légitime du révisionnisme, les historiens réexaminent de près des idées admises et les adaptent en fonction de nouvelles sources ou perspectives ; sous cette forme, le révisionnisme constitue alors une part importante du métier d'historien. L'historiographie post-moderne part en effet du principe qu'il n'existe pas Une Vérité : chaque description ou analyse d'une réalité historique est une construction élaborée par l'historien à partir de sources et d'une critique historique sérieuse. Il convient de revoir cette construction lorsque d'autres sources ou d'autres conceptions entrent en ligne de compte. Ce débat scientifique favorise une évolution de la conception de la réalité historique telle qu'elle $a p u$ être. Les 
Pourquoi les négationnistes restent dangereux (suite)
(2) Par exemple: Pourquoi le Révisionnisme de l'Holocauste? sur http://vho.org/Intro/F/index. html, consulté le 22 octobre 2015. historiens ne sont pas non plus unanimes quant aux détails de la Shoah : le nombre précis de victimes, l'instant précis où a été prise la décision de l'extermination, etc., sont sujets à débat.

Les négationnistes reprennent le terme de « révisionnisme» pour qualifier leurs intentions. De nombreux sites Internet ou publications négationnistes s'ouvrent donc aussi sur une analyse historico-scientifique de la nécessité du révisionnisme. Une analyse légitime, qu’ils réduisent aussitôt à la « révision » de la Shoah. Les négationnistes insistent sur le fait qu'une vision (lire : la leur) est tout aussi bonne que l'autre (celle scientifiquement acceptée) (Lipstadt, 1994, p. 17-18).

Mais en dépit de leurs allégations, la méthode utilisée par les négationnistes dans leur argumentation n'est nullement une forme légitime de révisionnisme. Ils partent en effet du principe que la Shoah n'a pas eu lieu (avec préméditation ou dans cette ampleur) et ajustent les faits à cette position. Un révisionnisme légitime implique l'apport de nouvelles sources et perspectives dans un débat existant, sans rejeter les sources précédentes ; de nouvelles interprétations historiques valables émergent ainsi aussi concernant la Shoah. Mais le révisionnisme négationniste pratique un raisonnement sélectif et choisit uniquement des sources qui étayent les conclusions fixées à l'avance. De cette manière, ils inversent complètement la méthode de recherche, d'analyse et de critique historique (McFee).

\section{LE NÉGATIONNISME COMME (PSEUDO-) SCIENCE}

Les négationnistes s'attribuent une image pseudoscientifique pour pouvoir se positionner comme alternative univalente à la science historique. Ils utilisent pour cela leurs propres canaux et réseaux qui imitent la démarche scientifique. La plus grande association de négationnistes aux États-Unis s'appelle l'Institute for Historical Review (Institut de révision historique) ; le mot Institute doit lui prêter une crédibilité scientifique et les termes Historical Review font volontairement supposer un champ d'investigation plus large que la Shoah, ce qui n'est pas le cas. L'organisation a en outre une revue «scientifique » au nom très innocent et attirant de Journal of Historical Review (Journal de révision historique). Un titre qui ne dépare pas et sème parfois la confusion avec de vraies revues historiques comme The American Historical Review (Revue d'histoire américaine) ou The Journal of Social History (Revue d'histoire sociale). L'association flamande «Vrij Historisch Onderzoek» créée par Siegfried Verbeke utilise également un nom trompeur pour une organisation négationniste. Le langage employé sur son site Internet et dans d'autres publications négationnistes est de même pseudoscientifique ; il semble objectif et commence par exposer des faits et constats que ne peut quapprouver toute personne rationnelle. Puis le texte s'éloigne peu à peu, et toujours plus, du discours scientifique usuel et aboutit aux théories de conspiration, à l'approbation des idées nazies et à une contestation ou une grossière minimalisation de l'extermination des Juifs ${ }^{2}$. 
Les négationnistes ne se contentent pas de diffuser leurs idées dans leur propre cercle. Aux États-Unis, ils sont invités à donner des conférences et des cours, et bénéficient même d'une tribune à la télévision (Lipstadt, 1994, p. 2-4). Mais en France aussi, des professeurs des Universités de Lyon II et III se font remarquer dans les années 1970 par leurs discours et lectures négationnistes ; il ne s'agit plus ici de personnages radicaux en marge de la société, mais d'intellectuels à la crédibilité universitaire (Rousso, 2006, p. 67-88). En 1985, le Français Henri Roques soutient à l’Université de Nantes un doctorat de littérature comparée aux allures d'essai à contenu négationniste (Igounet, 2000, p. 272-276).

\section{DES « CASSEURS DE MYTHES » SANS ARGUMENT}

L’argumentation adoptée par les contestataires de la Shoah et les négationnistes, de prime abord acceptable, est aussi leur plus gros point faible ; plutôt que de s'intéresser à la persécution des Juifs dans son ensemble, ils ne relèvent que quelques éléments sur lesquels ils ouvrent ensuite le feu. Les négationnistes espèrent alors qu'en exposant suffisamment un élément et en semant suffisamment le doute dessus, tout le récit perdra de sa crédibilité. En répétant suffisamment ce processus et en semant suffisamment le doute sur l'existence des chambres à gaz, il n'y a qu'un pas vers la négation d'un plan à l'origine de l'extermination. Ils suivent l'argumentation suivante : «Si un aspect est faux, pourquoi toute l'histoire ne serait-elle pas fausse ?» Ils espèrent, en faisant trembler chaque fondement, que le tableau s'effondrera comme un château de cartes.

En général, les négationnistes n'apportent pas beaucoup d'arguments personnels ; ils cherchent surtout à contester et affaiblir les arguments et les preuves de la science historique, des témoins et des sources. En semant suffisamment le doute sur le point de vue de son opposant, nul besoin d'avoir un point de vue propre. Ils sèment le doute en se présentant comme des « casseurs de mythes » et en insinuant des théories du complot qui éveillent chez les lecteurs ou les auditeurs une méfiance très difficile à réfuter.

\section{DES SOURCES SUSPECTES}

Les négationnistes essaient d'étouffer les arguments des historiens essentiellement en rendant suspectes les sources sur lesquelles ils s'appuient (Vanermen, 1996, p. $23,26,50$ ). Ils adoptent une attitude hypercritique en n'accordant pratiquement aucun crédit aux sources historiques (Rousso, 2006, p. 75). Les témoignages de victimes sont rejetés au titre de conspiration juive et d'exploitation politique de 
Pourquoi les négationnistes restent dangereux (suite) la Shoah. La confusion, les «échanges » ou la méconnaissance du quotidien précis dans les camps donnent parfois lieu à des contradictions ou des imprécisions dans les témoignages des victimes et à des suppositions. Les négationnistes utilisent ces imprécisions pour estampille mensonger et rejeter tout ce témoignage. Les témoignages de coupables sont à leur tour mis en doute, la plupart ayant été collectés juste après la guerre par les vainqueurs et ne pouvant donc être objectifs. Ici aussi, les théories du complot jouent un rôle. Citons pour exemple de ce raisonnement le témoignage de Rudolf Höss, commandant d’Auschwitz de 1940 à 1943. À son procès, il a amplement détaillé le processus d'extermination et la procédure de gazage. Mais les négationnistes n'accordent aucun crédit à ce témoignage, en partant du principe que Höss avait été torturé par les Alliés pendant sa captivité et contraint de faire cette déposition. Le Français Robert Faurisson affirme avoir une preuve de ces tortures : une conversation au cours de laquelle Höss aurait dit : « J'ai effectivement reconnu avoir tué 2,5 millions de Juifs, mais j’aurais tout aussi bien pu avouer qu'il s'agissait de 5 millions. » (Vanermen, 1996, p. 24). Ce témoignage n'est, il faut bien le dire, pas étudié de façon hypercritique par les négationnistes.

Autre exemple de cette argumentation pseudo scientifique : les négationnistes occultent complètement le contexte des sources. Pour eux, seule la source compte : les sources sont complètement isolées, examinées à l'extrême, jusqu'à perdre toute signification. Les négationnistes laissent complètement de côté les liens entre les différentes sources et le contexte général. Le Journal d'Anne Frank a également atterri sur la table d'examen du négationniste Robert Faurisson. Il a démontré que le journal était un faux, ayant constaté, "après examen critique approfondi », la présence de plusieurs écritures et styles, et l'absence ou l'ajout de certains passages dans les différentes éditions. Que ces styles différents s'expliquent par le fait qu’Anne avait elle-même réécrit des passages parce qu’elle espérait pouvoir les publier après la guerre, et que les passages manquant dans les premières éditions étaient ceux que son père trouvait trop intimes pour les publier (mais qui furent pourtant ajoutés par la suite), appartient à l'analyse contextuelle et dépasse l'analyse de Faurisson (Vanermen, 1996, p. 52-57).

Les historiens négationnistes essaient également de saper les sources scientifiques positives. En 1988, dans le cadre d'une étude «scientifique», Fred Leuchter, un ingénieur américain concepteur d'installations d'exécution, a visitéles chambres à gaz d'Auschwitz et de Majdanek. Pour mener à bien cette étude, il a prélevé des échantillons de ciment dans les chambres à gaz pour tester la présence de matières toxiques. Il en a conclu que l'on trouvait plus de traces de cyanure et de Zyklon B dans les chambres de désinfection que dans les chambres à gaz et que, pour cette raison, personne ne pouvait avoir été gazé dans les chambres à gaz. L’historien britannique et négationniste David Irving était, selon ses propres dires, convaincu que l'histoire devait s'appuyer davantage sur des méthodes d'investigation scientifique exactes et moins sur les sources historiques traditionnelles «propres à l’interprétation » 
(Vanermen, 1996, p. 48 et Eatwell, 1991). En 1993, fut établi un rapport similaire qui, avec la même méthode, tire les mêmes conclusions (Vanermen, 1996, p. 49).

\section{CONCLUSION}

Par cette approche pseudoscientifique qui les pousse à considérer leur avis au moins aussi valable que l'historiographie scientifique, les négationnistes parviennent à semer le doute tant sur l'ampleur que sur la planification de l'extermination des Juifs. Si en Belgique, les déclarations révisionnistes publiques sont rares et font la plupart du temps l'objet de poursuites, Internet offre une source presque inépuisable de sites et de textes révisionnistes. Malgré la législation, on tombe également facilement en Belgique sur des écrits révisionnistes (on peut par exemple les commander librement sur Amazon). En revendiquant leur caractère scientifique, ces textes en accès libre sont beaucoup plus dangereux que des allocutions ou discussions provocantes dans la sphère publique. Cela constitue d'une part un danger pour l'historiographie objective, d'autre part un danger éthique. Si le doute semé sur la Shoah devient suffisamment fort, cela ouvrira en effet la porte à la justification du nazisme et des idées (néo-) nazies, et à leur acceptation. I

Traduit du néerlandais par Emilie Syssau

\section{BIBLIOGRAPHIE}

- Bilefsky, Dan, « EU adopts measure outlawing Holocauste denial », The New York Times, 19 avril 2007.

- Brinckman, Bart, «Vlaamse peetvader moet naar Duitse cel », De Standaard, 29 octobre 2005, p. 4.

- Eatwell, Roger (1991) : «The Holocauste Denial: A Study in Propaganda Technique », in Cheles, L. (dir.) Neo-Fascism in Europe, New York, Longman, p. 120-146.

- Igounet, Valérie (2000) : Histoire du négationnisme en France, Paris, Seuil.

- Lechtholz-Zey, Jacqueline, Laws Banning Holocauste Denial, sur http://www.génocidepreventionnow.org (consulté le 21 octobre 2015).

- Lipstadt, Deborah (1994) : Denying the Holocauste, the growing assault on thruth and memory, Londres, Penguin.

- McFee, Gordon, «Why “Revisionism” Isn't », The Holocauste History Project,

http://www.Holocauste-history.org/revisionism-isnt/ (consulté le 12 octobre 2015).

- Rousso, Henry et al., "The Political and Cultural Roots of Negationism in France », in South Central Review, vol. 23, n 1, Fascism, Nazism: Cultural Legacies of Reaction (Spring, 2006), p. 67-88.

- s.n. Pourquoi le Révisionnisme de l'Holocauste? sur http://vho.org/Intro/F/index (consulté le 22 octobre 2015).

- Shermer, Michael et Grobman, Alex (2000) : Denying history, who says the Holocaust never happened and why do they say it? Berkeley, University of California Press.

- Georges, Timmerman, « De lange carrière van Siegfried Verbeke », De Morgen, 15 avril 2005, p. 4.

- Vanermen, Stijn (1996) : De ontkenning van de Jodenuitroeiing, Bruxelles, VUB Press.

- Vrielink, Jochum (2010) : Van haat gesproken? Een rechtsantropologisch onderzoek naar de bestrijding van rasgerelateerde uitingsdelicten in België, Anvers, Maklu.

- Widmann, Richard, Profiles in history: Paul Rassinier, sur Inconvenient History, 2010,

http://www.inconvenienthistory.com (consulté le 23 octobre 2015). Traduction de la citation : Emilie Syssau. 